Pol J Med Phys Eng 2012;18(2):41-47.

PL ISSN 1425-4689

doi: 10.2478/v10013-012-0006-y

website: http://versita.com/pjmpe

\author{
Tomasz Pliszczyński ${ }^{1}$, Katarzyna Ciszewska ${ }^{1}$, Małgorzata Dymecka ${ }^{1}$, \\ Jakub Ośko $^{1}$, Zbigniew Haratym ${ }^{1}$
}

\title{
Assessment of occupational internal exposure to beta emitters from the nuclear reactor primary coolant circuit
}

\author{
${ }^{1}$ National Centre for Nuclear Research, Otwock-Świerk, Sołtana 7, Poland \\ e-mail: tomasz.pliszczynski@ncbj.gov.pl
}

\begin{abstract}
Fission products of ${ }^{235} \mathrm{U}$ or isotopes from activation may appear in technological waters at normal operation of a research reactor. Therefore, reactor cooling water may contain a number of beta radioactive isotopes including yttrium and strontium isotopes, which can pose potential hazard to reactor personnel. In order to asses internal exposure urinalysis is carried out. This work presents the method of sample preparation and measurement used by Radiation Protection Measurements Laboratory (RPLM) of the National Centre for Nuclear Research (NCNR). Method of various isotopes of yttrium and Sr-90 activity calculation is also shown. Determination of these isotopes activities in urine sample allows estimating the effective doses. Key words: yttrium, strontium, urine, occupational exposure, effective dose
\end{abstract}

\section{Introduction}

A number of beta radioactive isotopes may be present in reactor cooling water. Sr-90 is one of the most important elements from the point of view of internal exposure while it has a long physical and biological half-life and it accumulates in bones. Furthermore, its daughter product Y-90 (Sr-90 and Y-90 remain in equilibrium) emits high-energy beta radiation, which can cause the damage to bone marrow [5].

In order to assess the exposure, measurements of Sr-90 activity are carried out using in vitro method by urinalysis of an exposed person. That kind of measurement does not cause any problems, provided that no other beta emitting isotopes of strontium and yttrium are present in the urine at the same time. In the case of contamination with reactor cooling water such assumption is false. Radioisotopes of strontium and yttrium are formed as a result of fission of U-235 in the fuel. They penetrate into the water because of the leakage of fuel elements or due to nuclear fission of U-235 which can be present on the fuel elements surface [1]. Efficiencies with which various isotopes of strontium and yttrium are formed are similar (Sr-89 - 4.8\%; Sr-90+Y-90 - 5.9\% ; Y-91 - 5.9\%) [4]. These isotopes are potentially hazardous to personnel repairing 
all types of equipment associated with the reactor cooling system. Dose derived from internal contamination with $\mathrm{Sr}-90$ is 10 times higher than those from yttrium isotopes or Sr-89.

There has been many methods of Sr-90 determination developed over the years. A method used in RPML-NCBJ (Radiation Protection Measurements Laboratory of National Centre for Nuclear Research), based on liquid-liquid extraction of Y-90 and indirect determination of Sr-90 content by the measurement of Y-90, is one of them [2].

Another widely used method is based on the separation of Sr-90 using extraction chromatography. This method, unlike the previous one, requires waiting (usually about 14 days) for Y-90 ingrowth and establishment of equilibrium between Sr-90 and Y-90. A non-radiometric method - mass spectrometry can be also named as the one which enables to determine Sr-90 content. It is a technique which allows separating and sorting the components of the sample according to their mass-to-charge ratio. As a result of using the first mentioned above method, a mixture of Y-90 and Y-91 will be obtained after extraction. Assuming the use of the second method, the final sample will contain Sr-89 and Sr-90 which will generate Y-90. The use of mass spectrometer will allow distinguishing different isotopes of strontium and yttrium by their mass-to-charge ratio.

Obviously all of the above-mentioned methods create difficulties in measurement and interpretation. In this paper we will present the procedure used by RPLM based on the extraction of yttrium.

\section{Methods}

The workers, who are exposed to strontium and yttrium isotopes, are asked to collect twenty-four hour urine sample, which should be provided to laboratory. In order to isolate and determine strontium and yttrium isotopes the radiochemical procedure is carried out. The method is based on assumption that Sr-90 is in equilibrium with Y-90. All isotopes of strontium and yttrium are coprecipitated with calcium oxalates. Addition of calcium and strontium carrier and acid environment supports the process of coprecipitation. Obtained precipitate is mineralized with $65 \%$ nitric acid and then dissolved in diluted hydrochloric acid. The sample is submitted to extraction with bis(2-ethylhexyl)-orthophosphoric acid (HDEHP) in toluene in presence of yttrium carrier. Yttrium isotopes are retained by HDEHP, strontium isotopes remain in water phase. Reextraction of yttrium from organic phase to water phase is carried out using diluted nitric acid. Depending on the method of measurement, obtained extract is mixed with liquid scintillation cocktail or evaporated on the counting disc. The activity of prepared sample is measured in (LSC) Liquid Scintillation Counter (Perkin Elmer 2900 TR) or in gas proportional counter (Berthold LB510). The method of urine sample treatment is shown in Figure 1. 


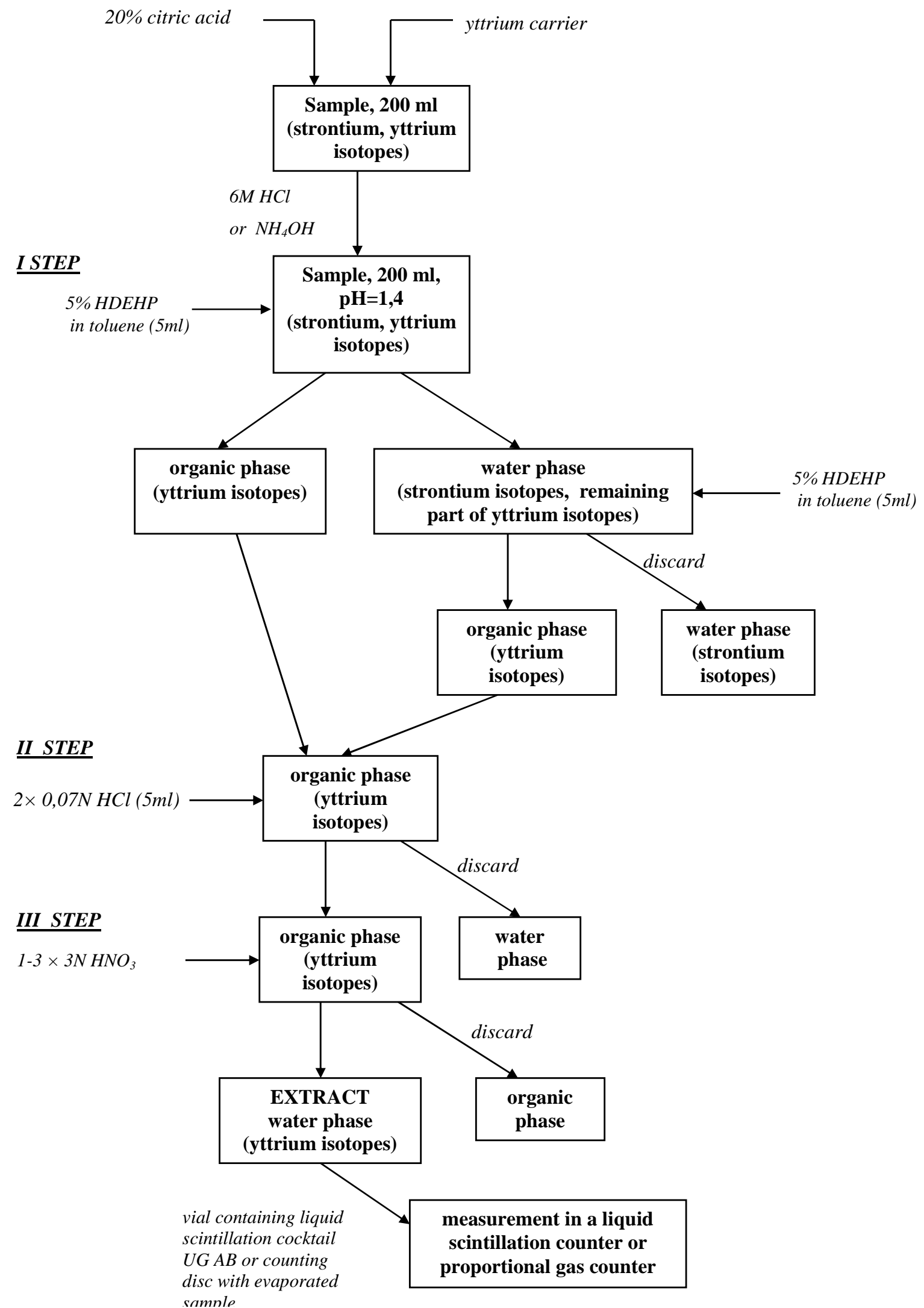

Figure 1. Extraction of ${ }^{90} \mathrm{Y}$ from urine sample 


\section{Results}

For the purpose of this type of analysis, liquid scintillation counter (LSC) is applied to assess the activity of yttrium isotopes. The solution measured in LSC consists of $17 \mathrm{ml}$ of liquid scintillation cocktail and $3 \mathrm{ml}$ of the sample.

After a single measurement, which is carried out at time $t_{1}$, the sum of activities ( $\left.A_{(O) 1}\right)$ of Y-90 and Y-91 can be determined. In order to assess the activity of individual isotopes, at least one additional measurement of total activity $\left(A_{Y}\right)$ ) should be carried out at the time $t_{2}$. The value of $t_{2}$ can not be lower than one half- life of Y-90 $\left(T_{1 / 2}=2.6\right.$ days $)$. This data allows calculating the activity of Y-90 and Y-91. Changes of total activity of Y-90+Y-91 and each component are shown in Figure 2.

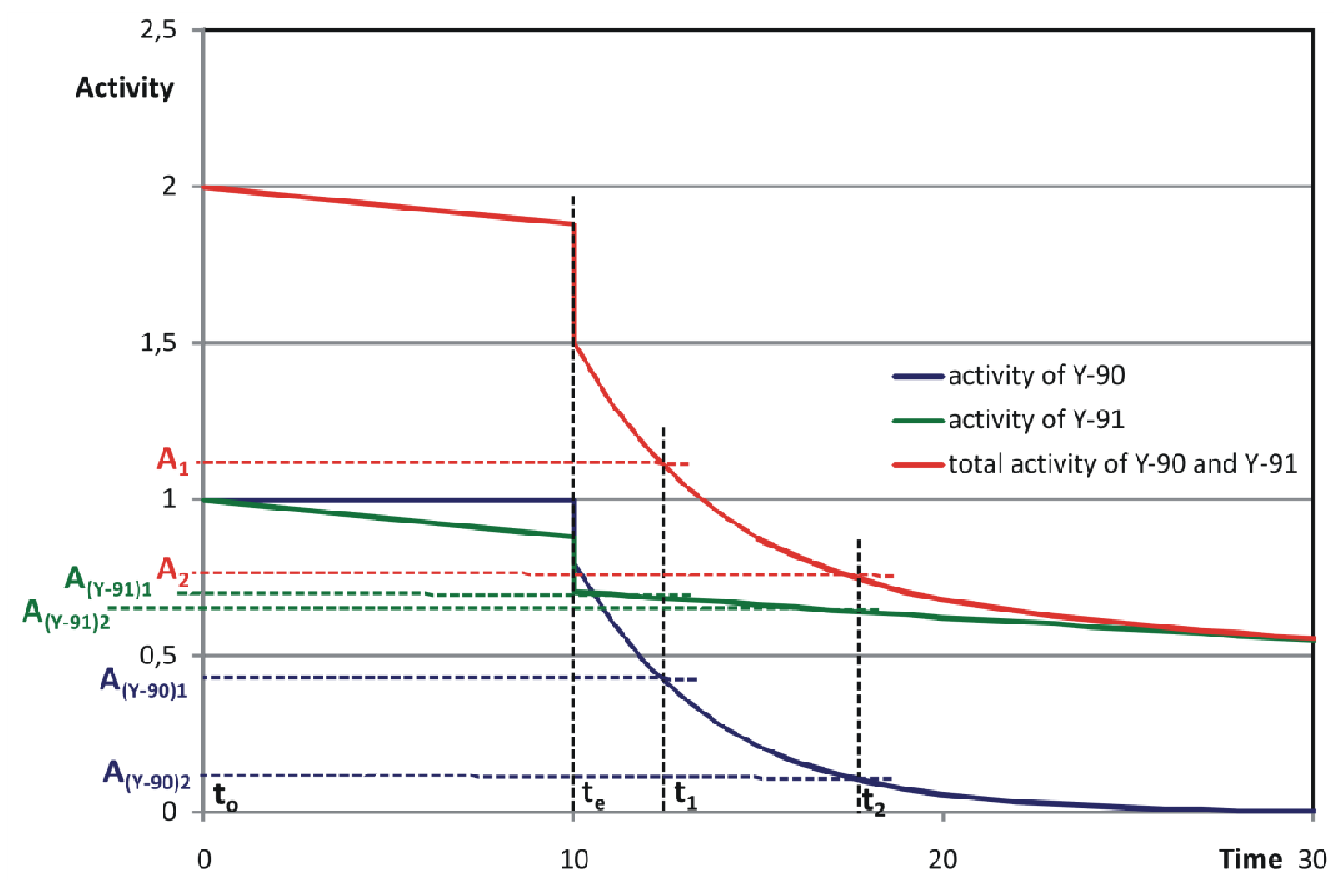

Figure 2. Changes of Y-90 and Y-91 activities in urine sample in the time between sample collection and measurement

Activity components are calculated as follows:

$A_{(r) 1}=A_{(\gamma-q) 1}+A_{(\gamma-q) 1}$

and

$A_{(\gamma) 2}=A_{(\gamma-91) 2}+A_{(\gamma-90) 2}$

Where $A_{1}$ and $A_{2}$ are activities of the sample calculated from data obtained from the measurements carried out at the time $t_{1}$ and $t_{2}$. 
Since:

$$
\begin{aligned}
& A_{(Y-91) 2}=A_{(Y-91) 1} \times \exp \left(\frac{-0,693 \cdot\left(t_{2}-t_{1}\right)}{\frac{T_{(Y-91) 1}}{2}}\right) \\
& A_{(Y-90) 2}=A_{(Y-90) 1} \times \exp \left(\frac{-0,693 \cdot\left(t_{2}-t_{1}\right)}{\frac{T_{(Y-90) 1}}{2}}\right)
\end{aligned}
$$

where:

$A_{(Y-90) 1}-$ activity of $Y-90$ isotope at $t_{1}[B q]$,

$A_{(Y-90) 2}-$ activity of $Y-90$ isotope at $t_{2}$ [Bq],

$A_{(Y-91) 1}$ - activity of $Y-91$ isotope at $t_{1}[B q]$,

$A_{(Y-91) z}$ - activity of $Y-91$ isotope at $t_{2}$ [Bq],

$\frac{T_{(Y-90) 1}}{3}$ - half-life of the isotope Y-90 [days],

$\frac{T_{(Y-91) 1}}{2}$ - half-life of the isotope Y-91[days].

after transformations we obtain:

$$
\begin{aligned}
& A_{W-90\rangle_{1}}=\frac{A_{(Y) 1}-A_{(Y) 2}}{1-\exp \left(\frac{-0,693\left(t_{2}-t_{2}\right)}{\frac{T(Y-90) 1}{2}}\right)} \\
& A_{W-91)_{1}}=\frac{A_{(Y) 1}-A_{W 2}}{1-\exp \left(\frac{-0.693\left(t_{2}-t_{1}\right)}{\frac{T(\gamma-91) 1}{2}}\right)}
\end{aligned}
$$

In order to increase the precision of estimation, the measurement can be repeated in subsequent time intervals which shall not, however, be shorter than $\frac{T_{(\gamma-90) 1}}{\mathbf{z}}$.

\section{Discussion}

The first step for determination on intake activity is calculating the Y-90 and Y-91 activity in the measured sample shortly before extraction. For this purpose activity determined for $t_{1}$ time, $A_{(\mathbb{W - 9 0 3}}$ and $A_{(Y-91)_{1}}$, must be converted to the time when extraction was started $t_{e}$, taking into account the chemical efficiency $\varepsilon$ of the procedure. The result of this calculation is Y-90 and Y-91 activity in the urine sample immediately before extraction.

It is important to note that the activity of Y-90 and Sr-90 remain in equilibrium with each other to the extraction beginning. For assessment of activity excreted with urine the Sr-90 + Y-90 and Y-91 radioactive decay between the time of sample collection 
(in routine procedures it is middle of collection period) and time when extraction started must be considered. The resulting equations are as follows:

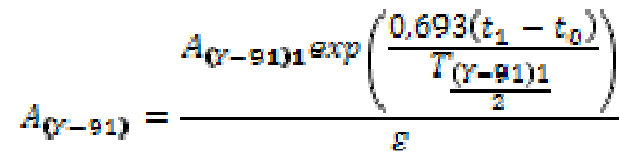

and

$A_{(s r-90)}=\frac{A_{(V-90) 1}}{z} \exp \left(\frac{0,693\left(t_{1}-t_{s}\right)}{\frac{T_{(\gamma-90) 1}}{2}}\right) \exp \left(\frac{0,693\left(t_{s}-t_{0}\right)}{\frac{T_{(s r-90) 1}}{2}}\right)$

where:

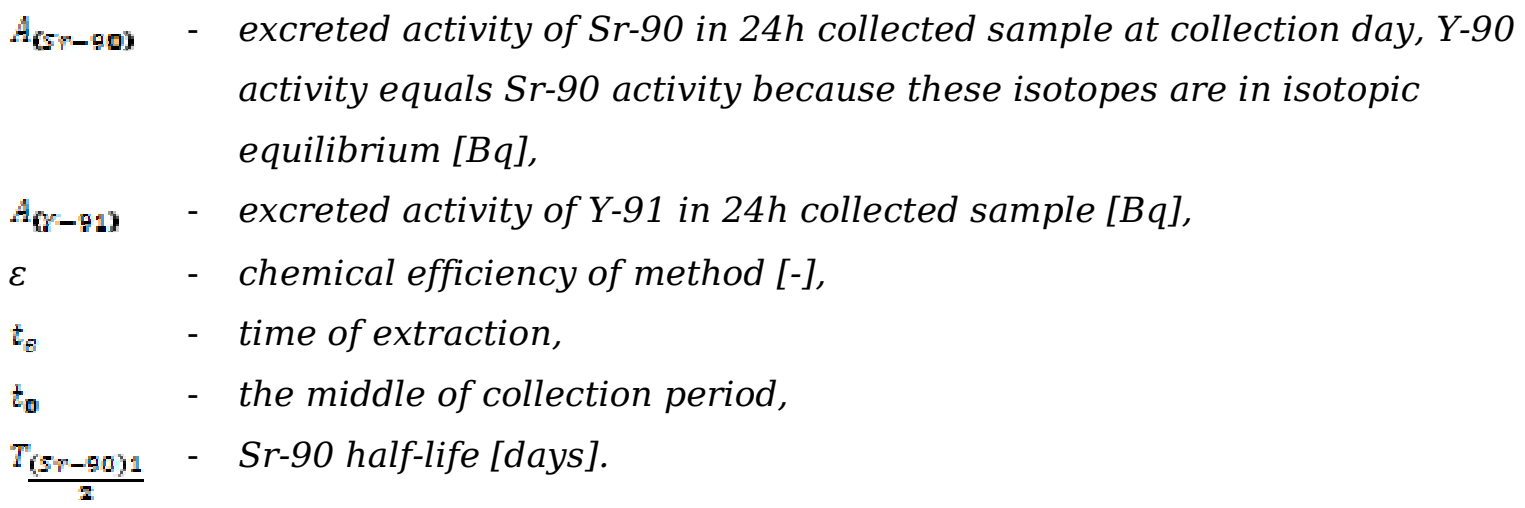

Determination of isotopes activity excreted with urine is the first step to the exposure assessment. In the next step the contamination scenario must be defined that is the date of intake, the route of intake, chemical form of radionuclide if it is possible and, additionally in case of inhalation, AMAD - Activity Median Aerodynamic Diameter of the aerosol particles in inhaled air [3].

This set of data combined with metabolism and retention/excretion model (according to ICRP models), and effective dose coefficient, e(g), allow to estimate the total committed effective dose.

\section{Conclusions}

The proposed procedure is relatively simple, inexpensive and rapid method for assessing the exposure coming from contamination with various yttrium and strontium isotopes. The result of estimates may be obtained after several days. The minimum detectable activity for this method is around $0,05 \mathrm{~Bq} / \mathrm{dm}^{3}$, therefore the effective dose at the level of several $\mu \mathrm{Sv}$ may be detected if the urine sample is collected some days after intake. The method accuracy is lower if the ratio of Y-91 activity to Sr-90 is higher than 20 and the assessment of Sr-90 activity is more difficult in this case. However, this is not a factor which disqualifies the method for radiation protection purposes because the Sr-90 contamination contributes to total committed effective dose only in $50 \%$. 


\section{References}

[1] G. Ackerman, Eksploatacja elektrowni jądrowych, Warszawa, WNT, 1987

[2] K. Ciszewska, T. Pliszczyński, M. Dymecka, J. Ośko, The improvement of the quality of effective doses estimation by interlaboratory comparisons, Pol J Med Phys Eng 2010;16(1):35-42

[3] IAEA, Indirect methods for assessing intakes of radionuclides causing ocupational exposure, IAEA, Safety Reports series No. 18.

[4] KAERI, Tables of radionuclides, Nuclear Data Center 2000 Korea Atomic Energy Research Institute, www.atom.kaeri.re.kr

[5] N. Vajda, C.K. Kim, Determination if radiostrontium isotopes: A review of analytical methodology, Appl Radiat Isotopes, 2010;68:2306-2326 